\title{
Time Trends in Sources and Dechlorination Pathways of Dioxins in Agrochemically Contaminated Sediments
} Minori Uchimiya* and Shigeki Masunaga

Graduate School of Environment and Information Sciences,

Yokohama National University, 79-7 Tokiwadai, Hodogaya, Yokohama 240-8501, Japan

uchimiya@ynu.ac.jp

\section{Revised manuscript submitted on February 5, 2007}

*Corresponding author fax: (510) 643-2940, phone: 510-643-9952, email: uchimiya@ynu.ac.jp.

Number of pages: 15

Number of Tables: 2

Number of figures: 6 


\section{Diagnostic Tools for the PMF Analysis}

The coefficient of determination (COD) for the PMF analyses of Lake Shinji and Tokyo Bay samples are given in Tables S1-2.

Table S1. The COD for Lake Shinji data set (83 chromatographic peaks and 12 samples).

Values below 0.60 are given in bold.

Number of Factors

\begin{tabular}{l|l|c|c|c|c|c}
\hline Homologue & Isomer & $\mathbf{1}$ & $\mathbf{2}$ & $\mathbf{3}$ & $\mathbf{4}$ & $\mathbf{5}$ \\
\hline TeCDD & 1368 & 0.75 & 0.94 & 0.96 & 0.99 & 0.98 \\
& 1379 & 0.74 & 0.94 & 0.95 & 0.98 & 0.98 \\
& 1369 & 0.84 & 0.90 & 0.94 & 0.99 & 0.99 \\
& $1247 / 1248 / 1378 / 1469$ & 0.81 & 0.88 & 0.99 & 0.99 & 0.99 \\
& $1246 / 1249 / 1268 / 1478$ & $\mathbf{0 . 0 4}$ & $\mathbf{0 . 2 1}$ & 0.65 & 0.94 & 0.94 \\
& 1279 & 0.77 & 0.79 & 0.90 & 0.99 & 0.99 \\
& $1234 / 1236 / 1269$ & 0.67 & 0.56 & 0.61 & 0.98 & 0.98 \\
& $1237 / 1238$ & 0.78 & 0.92 & 0.98 & 0.98 & 0.98 \\
& 2378 & $\mathbf{0 . 2 1}$ & $\mathbf{0 . 2 0}$ & 0.79 & 0.81 & 0.83 \\
& 1239 & 0.62 & 0.63 & 0.73 & 0.94 & 0.94 \\
& 1278 & $\mathbf{0 . 5 1}$ & $\mathbf{0 . 4 0}$ & $\mathbf{0 . 4 5}$ & 0.75 & 0.75 \\
& 1289 & 0.65 & $\mathbf{0 . 3 5}$ & $\mathbf{0 . 5 2}$ & 0.87 & 0.88 \\
\hline PeCDD & $12468 / 12479$ & 0.82 & 0.96 & 0.99 & 0.99 & 0.99 \\
& 12469 & $\mathbf{0 . 0 9}$ & $\mathbf{0 . 2 7}$ & 0.97 & 0.98 & 0.98 \\
& 12368 & 0.78 & 0.96 & 0.97 & 0.98 & 0.98 \\
& 12478 & $\mathbf{0 . 4 3}$ & $\mathbf{0 . 4 0}$ & 0.73 & 0.75 & 0.75 \\
& 12379 & 0.80 & 0.96 & 0.98 & 0.98 & 0.98 \\
& 12369 & $\mathbf{0 . 0 3}$ & $\mathbf{0 . 1 9}$ & 0.89 & 0.89 & 0.91 \\
& $12467 / 12489$ & $\mathbf{0 . 1 4}$ & $\mathbf{0 . 3 6}$ & 0.82 & 0.94 & 0.95 \\
& 12347 & 0.73 & 0.78 & 0.95 & 0.97 & 0.98 \\
& 12346 & $\mathbf{0 . 4 5}$ & 0.85 & 0.94 & 0.96 & 0.96 \\
& 12378 & 0.31 & 0.56 & 0.97 & 0.98 & 0.98 \\
& 12367 & 0.57 & 0.61 & 0.76 & 0.81 & 0.82 \\
& 12389 & $\mathbf{0 . 1 1}$ & 0.91 & 0.94 & 0.94 \\
\hline & $124679 / 124689$ & $\mathbf{0 . 0 0}$ & $\mathbf{0 . 0 8}$ & $\mathbf{0 . 3 8}$ & 0.75 & 0.82 \\
& 123468 & $\mathbf{0 . 3 6}$ & $\mathbf{0 . 5 3}$ & 0.82 & 0.98 & 0.98 \\
& $123679 / 123689$ & $\mathbf{0 . 2 7}$ & $\mathbf{0 . 4 1}$ & 0.71 & 0.78 & 0.94 \\
& 123469 & 0.59 & 0.60 & 0.74 & 0.77 & 0.82 \\
& 123478 & $\mathbf{0 . 2 7}$ & $\mathbf{0 . 2 3}$ & 0.78 & 0.79 & 0.89 \\
& 123678 & $\mathbf{0 . 3 3}$ & 0.69 & 0.84 & 0.86 & 0.87 \\
& $1,2,3,7,8,9$ & $\mathbf{0 . 4 9}$ & 0.71 & 0.75 & 0.80 & 0.91 \\
\hline \multirow{2}{*}{ HxCDD } & & & & & \\
& 123467 & 0.85 & 0.88 & 0.89 \\
\hline
\end{tabular}




\begin{tabular}{|c|c|c|c|c|c|c|}
\hline TeCDF & 1368 & 0.31 & 0.47 & 0.49 & 0.62 & 0.76 \\
\hline & 1468 & 0.61 & 0.75 & 0.75 & 0.89 & 0.91 \\
\hline & 2468 & 0.69 & 0.90 & 0.92 & 0.97 & 0.97 \\
\hline & $1247 / 1347 / 1378 / 1346 / 1246$ & 0.67 & 0.74 & 0.79 & 0.95 & 0.95 \\
\hline & $1367 / 1348 / 1379 / 1248$ & 0.69 & 0.72 & 0.74 & 0.89 & 0.90 \\
\hline & $1268 / 1467 / 1478$ & 0.58 & 0.63 & 0.63 & 0.86 & 0.87 \\
\hline & $1369 / 1237 / 2368$ & 0.78 & 0.90 & 0.91 & 0.97 & 0.98 \\
\hline & $2467 / 1238 / 1236 / 1469 / 1678 / 1234$ & 0.83 & 0.89 & 0.97 & 0.99 & 0.99 \\
\hline & 1278 & 0.40 & 0.36 & 0.48 & 0.76 & 0.83 \\
\hline & $1267 / 1349$ & 0.59 & 0.64 & 0.73 & 0.84 & 0.93 \\
\hline & $2,3,7,8$ & 0.11 & 0.42 & 0.59 & 0.64 & 0.88 \\
\hline & 2348/2347/2346/1249/1279 & 0.76 & 0.75 & 0.97 & 0.99 & 0.99 \\
\hline & 2367 & 0.50 & 0.37 & 0.37 & 0.82 & 0.82 \\
\hline & $3467 / 1269$ & 0.59 & 0.44 & 0.55 & 0.87 & 0.87 \\
\hline & 1239 & 0.12 & 0.14 & 0.20 & 0.30 & 0.33 \\
\hline & 1289 & 0.55 & 0.64 & 0.66 & 0.69 & 0.73 \\
\hline $\mathrm{PeCDF}$ & $13468 / 12468$ & 0.70 & 0.75 & 0.76 & 0.84 & 0.86 \\
\hline & 13678 & 0.73 & 0.70 & 0.94 & 0.97 & 0.97 \\
\hline & $12368 / 12478 / 13467 / 13478 / 12467$ & 0.81 & 0.92 & 0.95 & 0.98 & 0.98 \\
\hline & $13479 / 14678$ & 0.89 & 0.95 & 0.94 & 0.96 & 0.96 \\
\hline & 12479 & 0.42 & 0.59 & 0.81 & 0.83 & 0.79 \\
\hline & 13469 & 0.75 & 0.83 & 0.85 & 0.92 & 0.93 \\
\hline & $23468 / 12469 / 12347 / 12346$ & 0.79 & 0.96 & 0.98 & 0.99 & 0.99 \\
\hline & 12348 & 0.67 & 0.63 & 0.64 & 0.71 & 0.85 \\
\hline & 12378 & 0.61 & 0.60 & 0.97 & 0.96 & 0.96 \\
\hline & 12367 & 0.74 & 0.87 & 0.94 & 0.96 & 0.96 \\
\hline & $12678 / 12379$ & 0.80 & 0.86 & 0.96 & 0.98 & 0.98 \\
\hline & $2,3,4,7,8$ & 0.88 & 0.88 & 0.98 & 0.98 & 0.99 \\
\hline & $12489 / 12679 / 12369$ & 0.66 & 0.85 & 0.85 & 0.89 & 0.92 \\
\hline & 23467 & 0.86 & 0.81 & 0.93 & 0.98 & 0.99 \\
\hline & 12349 & 0.68 & 0.80 & 0.83 & 0.84 & 0.86 \\
\hline & 12389 & 0.68 & 0.62 & 0.67 & 0.71 & 0.78 \\
\hline $\mathrm{HxCDF}$ & 123468 & 0.30 & 0.40 & 0.99 & 0.99 & 0.99 \\
\hline & $134678 / 124678$ & 0.20 & 0.29 & 0.97 & 0.98 & 0.98 \\
\hline & 134679 & 0.67 & 0.83 & 0.89 & 0.89 & 0.89 \\
\hline & 124679 & 0.78 & 0.94 & 0.99 & 1.00 & 1.00 \\
\hline & 124689 & 0.00 & 0.00 & 0.95 & 0.95 & 0.97 \\
\hline & $123467 / 123478$ & 0.55 & 0.67 & 0.96 & 0.99 & 0.99 \\
\hline & 123678 & 0.55 & 0.73 & 0.94 & 0.95 & 0.95 \\
\hline & 123479 & 0.61 & 0.65 & 0.88 & 0.88 & 0.89 \\
\hline & $123469 / 123679$ & 0.29 & 0.33 & 0.85 & 0.86 & 0.88 \\
\hline & 123689 & 0.08 & 0.01 & 0.95 & 0.95 & 0.95 \\
\hline & 234678 & 0.85 & 0.98 & 0.98 & 0.99 & 0.99 \\
\hline & 123789 & 0.14 & 0.27 & 0.56 & 0.58 & 0.80 \\
\hline & 123489 & 0.73 & 0.80 & 0.80 & 0.91 & 0.91 \\
\hline $\mathrm{HpCDF}$ & 1234678 & 0.12 & 0.18 & 0.96 & 0.97 & 0.98 \\
\hline & 1234679 & 0.70 & 0.85 & 0.91 & 0.96 & 0.96 \\
\hline & 1234689 & 0.04 & 0.07 & 0.95 & 0.96 & 0.96 \\
\hline & 1234789 & 0.15 & 0.21 & 0.93 & 0.96 & 0.98 \\
\hline & OcCDF & 0.08 & 0.13 & 0.92 & 0.92 & 0.95 \\
\hline & OcCDD & 0.77 & 0.97 & 0.97 & 0.98 & 0.98 \\
\hline
\end{tabular}


Table S2. The COD for the Tokyo Bay core and surface sediment samples combined (83 chromatographic peaks and 23 samples). Values below 0.60 are given in bold.

\begin{tabular}{l|l|c|c|c|c|c}
\multicolumn{7}{c|}{ Number of Factors } \\
\hline Homologue & Isomer & $\mathbf{1}$ & $\mathbf{2}$ & $\mathbf{3}$ & $\mathbf{4}$ & $\mathbf{5}$ \\
\hline TeCDD & 1368 & $\mathbf{0 . 2 1}$ & $\mathbf{0 . 1 9}$ & $\mathbf{0 . 3 2}$ & 0.97 & 0.98 \\
& 1379 & $\mathbf{0 . 1 7}$ & $\mathbf{0 . 1 7}$ & $\mathbf{0 . 3 6}$ & 0.98 & 0.98 \\
& 1369 & $\mathbf{0 . 0 2}$ & $\mathbf{0 . 1 1}$ & $\mathbf{0 . 0 2}$ & 0.97 & 0.98 \\
& $1247 / 1248 / 1378 / 1469$ & $\mathbf{0 . 5 4}$ & 0.74 & 0.76 & 0.99 & 0.99 \\
& $1246 / 1249 / 1268 / 1478$ & 0.65 & 0.87 & 0.90 & 0.97 & 0.97 \\
& 1279 & 0.69 & 0.91 & 0.94 & 0.98 & 0.98 \\
& $1234 / 1236 / 1269$ & 0.57 & 0.69 & 0.71 & 0.84 & 0.86 \\
& $1237 / 1238$ & $\mathbf{0 . 0 0}$ & $\mathbf{0 . 0 3}$ & $\mathbf{0 . 4 3}$ & 0.95 & 0.96 \\
& 2378 & 0.69 & 0.90 & 0.93 & 0.96 & 0.96 \\
& 1239 & 0.73 & 0.89 & 0.90 & 0.97 & 0.97 \\
& 1278 & 0.74 & 0.95 & 0.98 & 0.99 & 0.99 \\
& 1289 & 0.62 & 0.85 & 0.92 & 0.94 & 0.96 \\
\hline PeCDD & $12468 / 12479$ & 0.58 & 0.64 & 0.71 & 0.99 & 0.99 \\
& 12469 & 0.74 & 0.94 & 0.97 & 0.98 & 0.99 \\
& 12368 & $\mathbf{0 . 1 0}$ & $\mathbf{0 . 1 3}$ & $\mathbf{0 . 3 9}$ & 0.98 & 0.98 \\
& 12478 & 0.77 & 0.95 & 0.97 & 0.98 & 0.98 \\
& 12379 & $\mathbf{0 . 0 0}$ & $\mathbf{0 . 0 0}$ & $\mathbf{0 . 2 6}$ & 0.97 & 0.97 \\
& 12369 & 0.60 & 0.65 & 0.65 & 0.65 & 0.84 \\
& $12467 / 12489$ & 0.75 & 0.95 & 0.98 & 0.98 & 0.98 \\
& 12347 & 0.80 & 0.92 & 0.92 & 0.97 & 0.98 \\
& 12346 & 0.73 & 0.92 & 0.94 & 0.96 & 0.98 \\
& 12378 & 0.85 & 0.98 & 0.99 & 0.99 & 0.99 \\
& 12367 & 0.86 & 0.96 & 0.96 & 0.97 & 0.99 \\
& 12389 & 0.75 & 0.91 & 0.93 & 0.93 & 0.97 \\
\hline HxCDD & $124679 / 124689$ & 0.74 & 0.94 & 0.97 & 0.98 & 0.98 \\
& 123468 & 0.83 & 0.94 & 0.94 & 0.97 & 0.97 \\
& $123679 / 123689$ & 0.91 & 0.95 & 0.96 & 0.95 & 0.96 \\
& 123469 & 0.78 & 0.82 & 0.82 & 0.82 & 0.92 \\
& 123478 & 0.95 & 0.98 & 0.98 & 0.99 & 0.99 \\
& 123678 & 0.82 & 0.83 & 0.84 & 0.85 & 0.93 \\
& 123789 & 0.77 & 0.95 & 0.98 & 0.98 & 0.98 \\
& 123467 & $\mathbf{0 . 2 3}$ & $\mathbf{0 . 2 2}$ & $\mathbf{0 . 4 9}$ & 0.65 & 0.83 \\
\hline & 1234679 & 0.76 & 0.85 & 0.91 & 0.91 & 0.91 \\
& 1234678 & $\mathbf{0 . 2 1}$ & 0.73 & 0.76 & 0.78 & 0.86 \\
\hline
\end{tabular}




\begin{tabular}{|c|c|c|c|c|c|c|}
\hline \multirow[t]{15}{*}{$\mathrm{TeCDF}$} & 1368 & 0.56 & 0.50 & 0.57 & 0.63 & 0.82 \\
\hline & 1468 & 0.90 & 0.94 & 0.94 & 0.95 & 0.95 \\
\hline & 2468 & 0.11 & 0.11 & 0.34 & 0.98 & 0.98 \\
\hline & $1247 / 1347 / 1378 / 1346 / 1246$ & 0.79 & 0.96 & 0.99 & 0.99 & 1.00 \\
\hline & $1367 / 1348 / 1379 / 1248$ & 0.81 & 0.94 & 0.95 & 0.96 & 0.96 \\
\hline & $1268 / 1467 / 1478$ & 0.84 & 0.96 & 0.97 & 0.98 & 0.99 \\
\hline & $1369 / 1237 / 2368$ & 0.83 & 0.96 & 0.97 & 0.98 & 0.98 \\
\hline & $2467 / 1238 / 1236 / 1469 / 1678 / 1234$ & 0.95 & 0.96 & 0.98 & 0.98 & 0.99 \\
\hline & 1278 & 0.58 & 0.85 & 0.97 & 0.99 & 0.99 \\
\hline & $1267 / 1349$ & 0.50 & 0.73 & 0.81 & 0.85 & 0.90 \\
\hline & $2348 / 2378 / 2347 / 2346 / 1249 / 1279$ & 0.67 & 0.91 & 0.98 & 0.99 & 1.00 \\
\hline & 2367 & 0.88 & 0.94 & 0.94 & 0.94 & 0.97 \\
\hline & $3467 / 1269$ & 0.95 & 0.92 & 0.95 & 0.97 & 0.97 \\
\hline & 1239 & 0.74 & 0.89 & 0.89 & 0.91 & 0.92 \\
\hline & 1289 & 0.45 & 0.63 & 0.75 & 0.76 & 0.93 \\
\hline \multirow[t]{15}{*}{$\overline{\mathrm{PeCDF}}$} & $13468 / 12468$ & 0.17 & 0.64 & 0.73 & 0.81 & 0.83 \\
\hline & 13678 & 0.93 & 0.85 & 0.96 & 0.98 & 0.98 \\
\hline & $12368 / 12478 / 13467 / 13478 / 12467$ & 0.95 & 0.99 & 0.99 & 0.99 & 0.99 \\
\hline & $13479 / 14678$ & 0.82 & 0.73 & 0.87 & 0.90 & 0.91 \\
\hline & 12479 & 0.84 & 0.71 & 0.86 & 0.94 & 0.95 \\
\hline & 13469 & 0.89 & 0.78 & 0.87 & 0.97 & 0.97 \\
\hline & $23468 / 12469 / 12347 / 12346$ & 0.48 & 0.47 & 0.94 & 0.95 & 0.95 \\
\hline & 12348 & 0.78 & 0.95 & 0.99 & 0.98 & 0.99 \\
\hline & 12378 & 0.63 & 0.88 & 0.98 & 0.99 & 0.99 \\
\hline & 12367 & 0.97 & 0.88 & 0.93 & 0.98 & 0.98 \\
\hline & $12678 / 12379$ & 0.87 & 0.99 & 1.00 & 1.00 & 1.00 \\
\hline & $23478 / 12489 / 12679 / 12369$ & 0.90 & 0.99 & 0.99 & 0.99 & 0.99 \\
\hline & 23467 & 0.72 & 0.58 & 0.90 & 0.97 & 0.97 \\
\hline & 12349 & 0.73 & 0.57 & 0.89 & 0.99 & 0.99 \\
\hline & 12389 & 0.80 & 0.96 & 0.98 & 0.98 & 0.98 \\
\hline \multirow[t]{14}{*}{$\mathrm{HxCDF}$} & 123468 & 0.61 & 0.66 & 0.68 & 0.69 & 0.88 \\
\hline & $134678 / 124678$ & 0.41 & 0.54 & 0.66 & 0.67 & 0.77 \\
\hline & 134679 & 0.86 & 0.75 & 0.89 & 0.90 & 0.98 \\
\hline & 124679 & 0.65 & 0.55 & 0.85 & 0.96 & 0.97 \\
\hline & 124689 & 0.55 & 0.86 & 0.87 & 0.88 & 0.87 \\
\hline & 123478 & 0.63 & 0.87 & 0.98 & 0.99 & 0.99 \\
\hline & 123467 & 0.44 & 0.39 & 0.73 & 0.90 & 0.91 \\
\hline & 123678 & 0.94 & 0.98 & 0.98 & 0.98 & 0.99 \\
\hline & 123479 & 0.95 & 0.95 & 0.97 & 0.97 & 0.97 \\
\hline & $123469 / 123679$ & 0.70 & 0.59 & 0.79 & 0.94 & 0.94 \\
\hline & 123689 & 0.95 & 0.92 & 0.95 & 0.96 & 0.98 \\
\hline & 234678 & 0.69 & 0.58 & 0.95 & 0.97 & 0.98 \\
\hline & 123789 & 0.55 & 0.69 & 0.72 & 0.72 & 0.79 \\
\hline & 123489 & 0.85 & 0.94 & 0.94 & 0.94 & 0.98 \\
\hline \multirow[t]{6}{*}{$\mathrm{HpCDF}$} & 1234678 & 0.39 & 0.41 & 0.61 & 0.67 & 0.79 \\
\hline & 1234679 & 0.60 & 0.48 & 0.78 & 0.96 & 0.97 \\
\hline & 1234689 & 0.64 & 0.91 & 0.96 & 0.96 & 0.96 \\
\hline & 1234789 & 0.68 & 0.70 & 0.75 & 0.89 & 0.91 \\
\hline & OcCDF & 0.01 & 0.00 & 0.55 & 0.55 & 0.70 \\
\hline & OcCDD & 0.04 & 0.01 & 0.77 & 0.79 & 0.87 \\
\hline
\end{tabular}




\section{Reference PCDD/F Congener Profiles for PCP, CNP, and Atmospheric Deposition.}

PCP. The dioxin byproducts formed during the chlorination of phenol to form PCP is dominated by heptachlorinated (1,2,3,4,6,7,9- and 1,2,3,4,6,7,8-substituted) homologues and $\operatorname{OcCDF}(1)$. It is hypothesized that heptachlorinated homologues are formed via the coupling between PCP and the major chlorination intermediate 2,3,4,6-tetrachlorophenol (1). The HpCDDs formed during the manufacturing of PCP is dominated by the 1,2,3,4,6,7,8-substituted congener (1). The OcCDF is formed by the coupling of two PCP molecules (1). Between 1958 and 1983, 164 ktons (national sum) of PCP was used as herbicide in rice paddies (2).

CNP. Signature TeCDD byproducts of CNP synthesis from 2,4,6-trichlorophenol and 4chloronitrobenzene are 1,3,6,8- and 1,3,7,9-TeCDDs (1). The primary, 1,3,6,8-substituted TeCDD is likely formed as a coupling product of two 2,4,6-trichlorophenols (1). The 1,3,7,9TeCDD can be formed by the coupling of two 2,4,6-trichlorophenols followed by the Smiles rearrangement(1). $\mathrm{CNP}$ was introduced as a replacement of $\mathrm{PCP}$ and was used in total of 78 ktons (national sum) between 1965 and 1994 (1).

Combustion. Congener profiles of atmospheric deposition in Kanto Region of Japan closely resembled that arising from the waste incineration processes (3). In Japan, emission of dioxins from municipal and industrial waste incineration plants continued to increase since 1960s, reaching maximum by 1997 (4). 


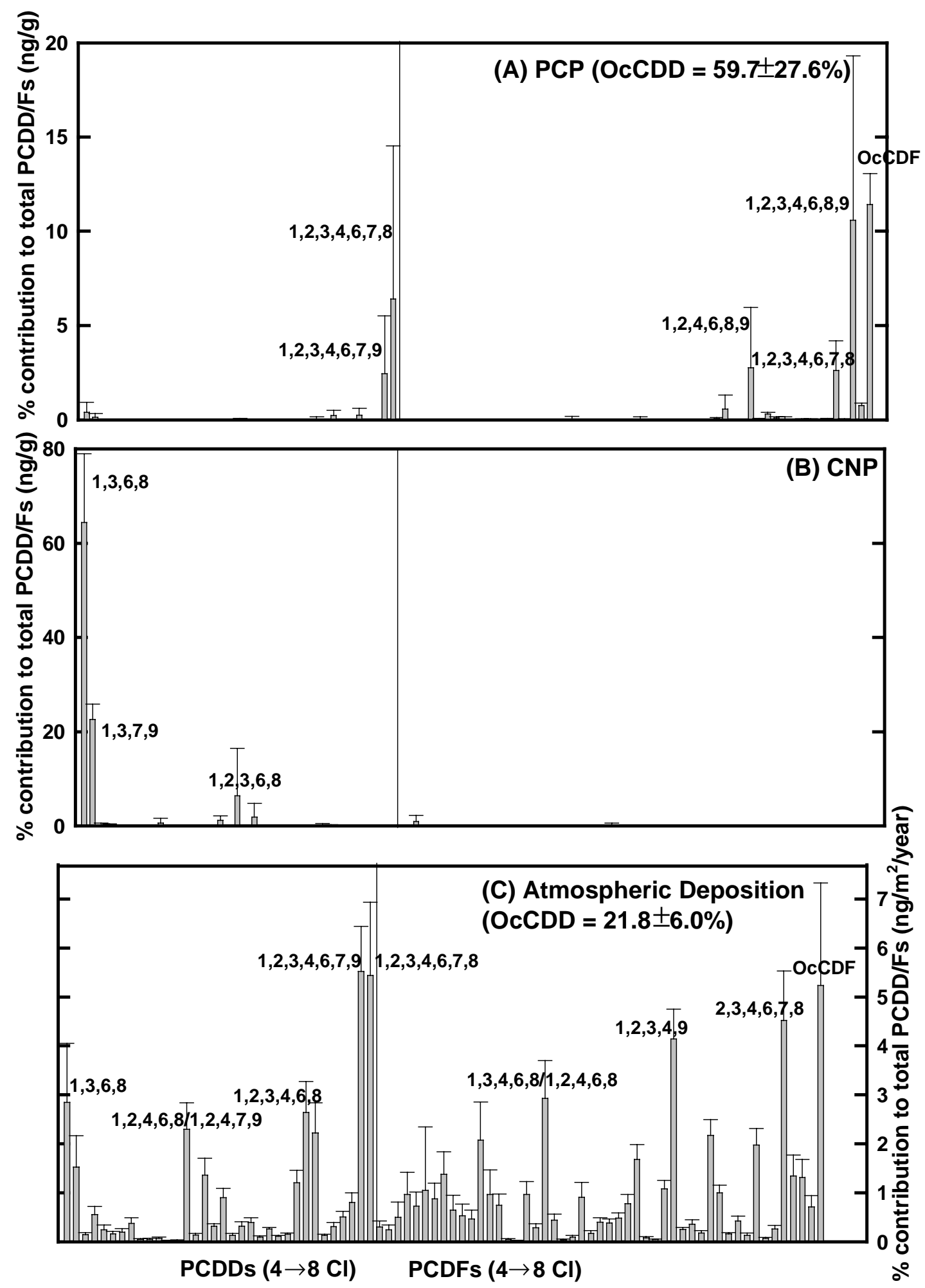

Figure S1. Reference PCDD/F congener profiles for (A) PCP manufactured in late 1960s ( $\mathrm{n}=2$ ) (1), (B) CNP manufactured in late 1970s $(\mathrm{n}=5)(1)$, and (C) atmospheric deposition in Yokohama and Tokyo $(\mathrm{n}=16)(5)$ in \% contribution to total PCDD/F concentrations (original units are given parentheses). The OcCDD was plotted only in (B). Error bars show standard deviation. 
III. Percent Factor Contributions of PMF-derived Fingerprints.

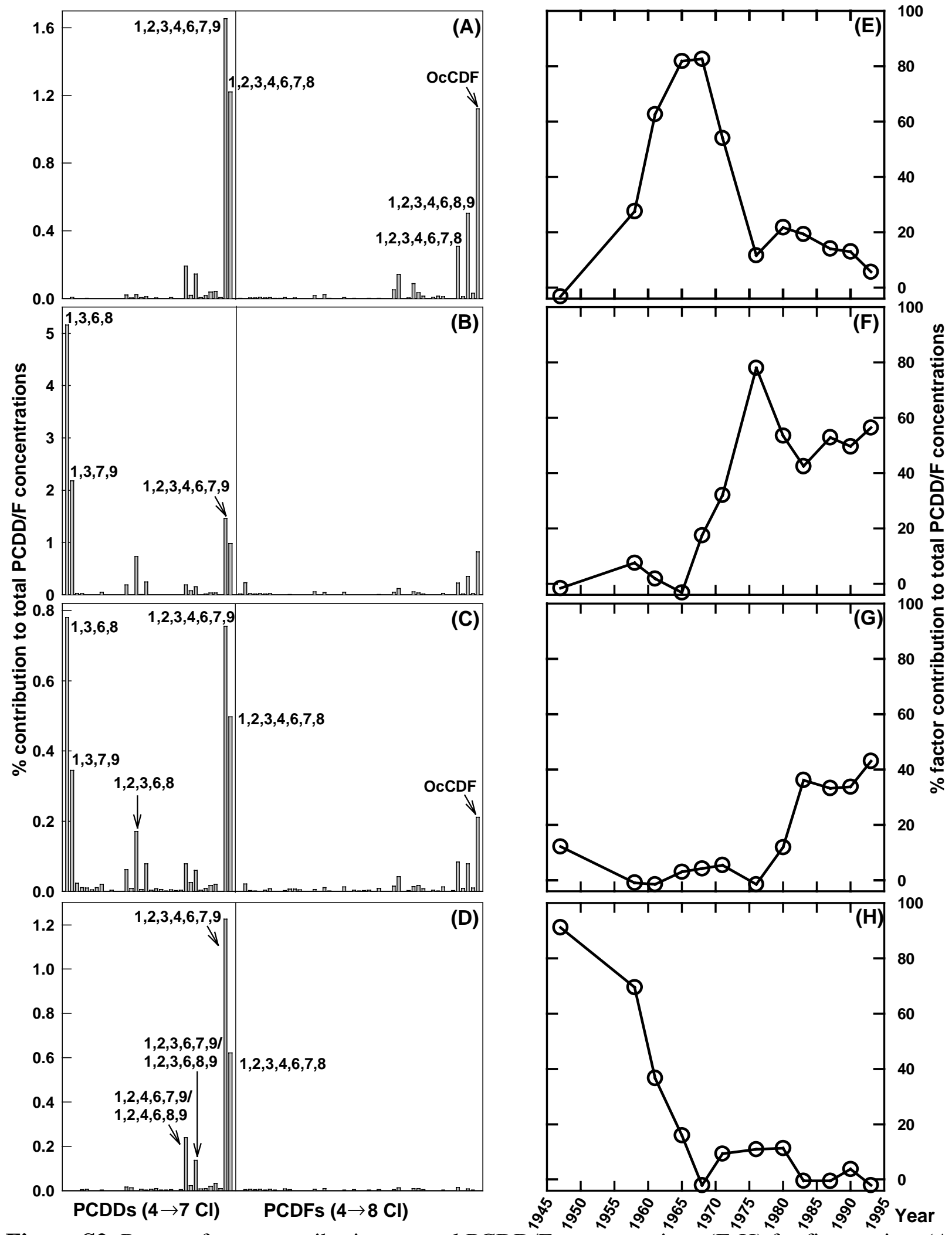

Figure S2. Percent factor contribution to total PCDD/F concentrations (E-H) for fingerprints (AD) derived from Lake Shinji data set. In A-D, vertical lines separate PCDDs and PCDFs. 

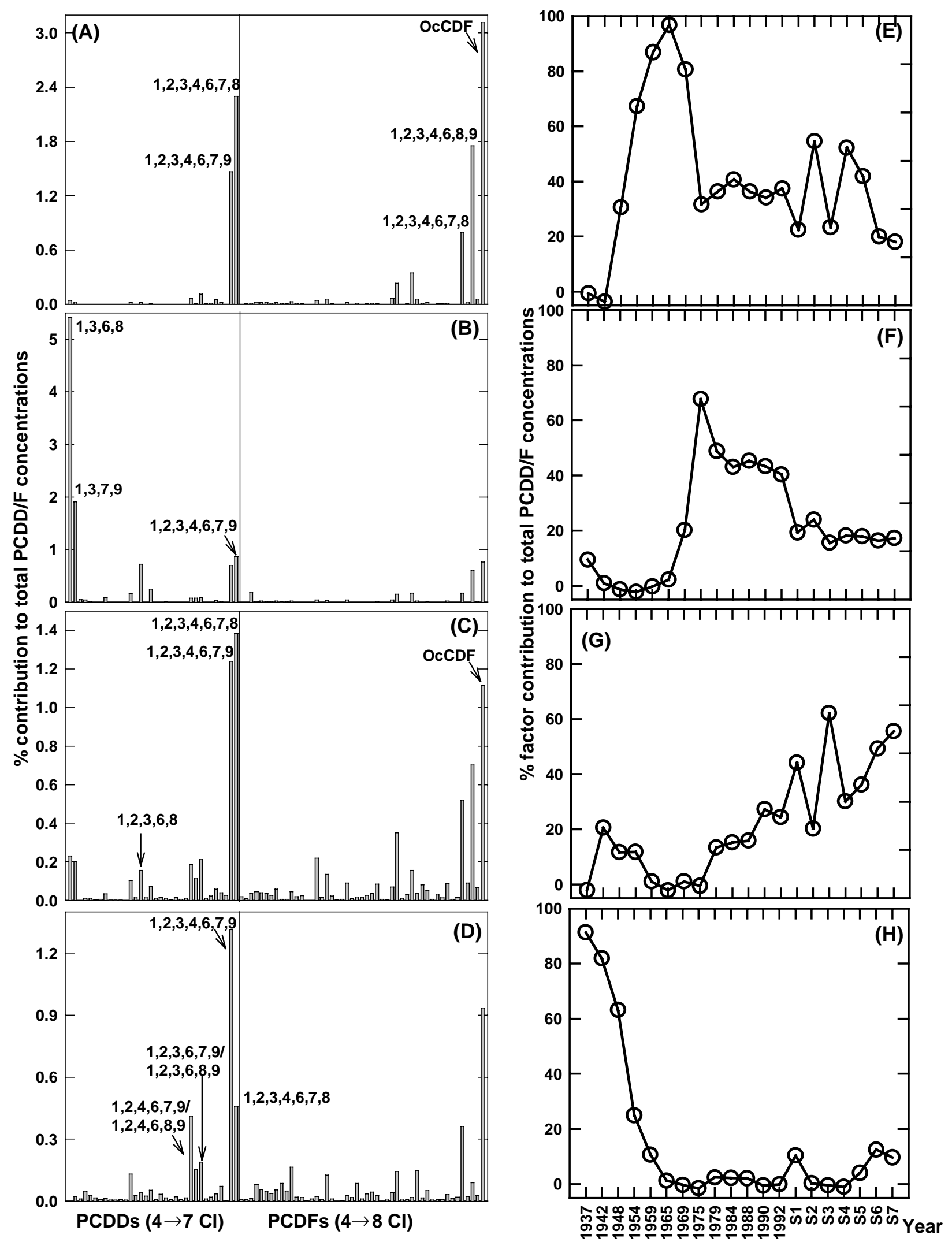

Figure S3. Percent factor contribution to total PCDD/F concentrations (E-H) for fingerprints (AD) derived from Tokyo Bay data set. S1-7 in E-H denote surface sediments. 
IV. Total Concentration of Each Homologue in Lake Shinji and Tokyo Bay
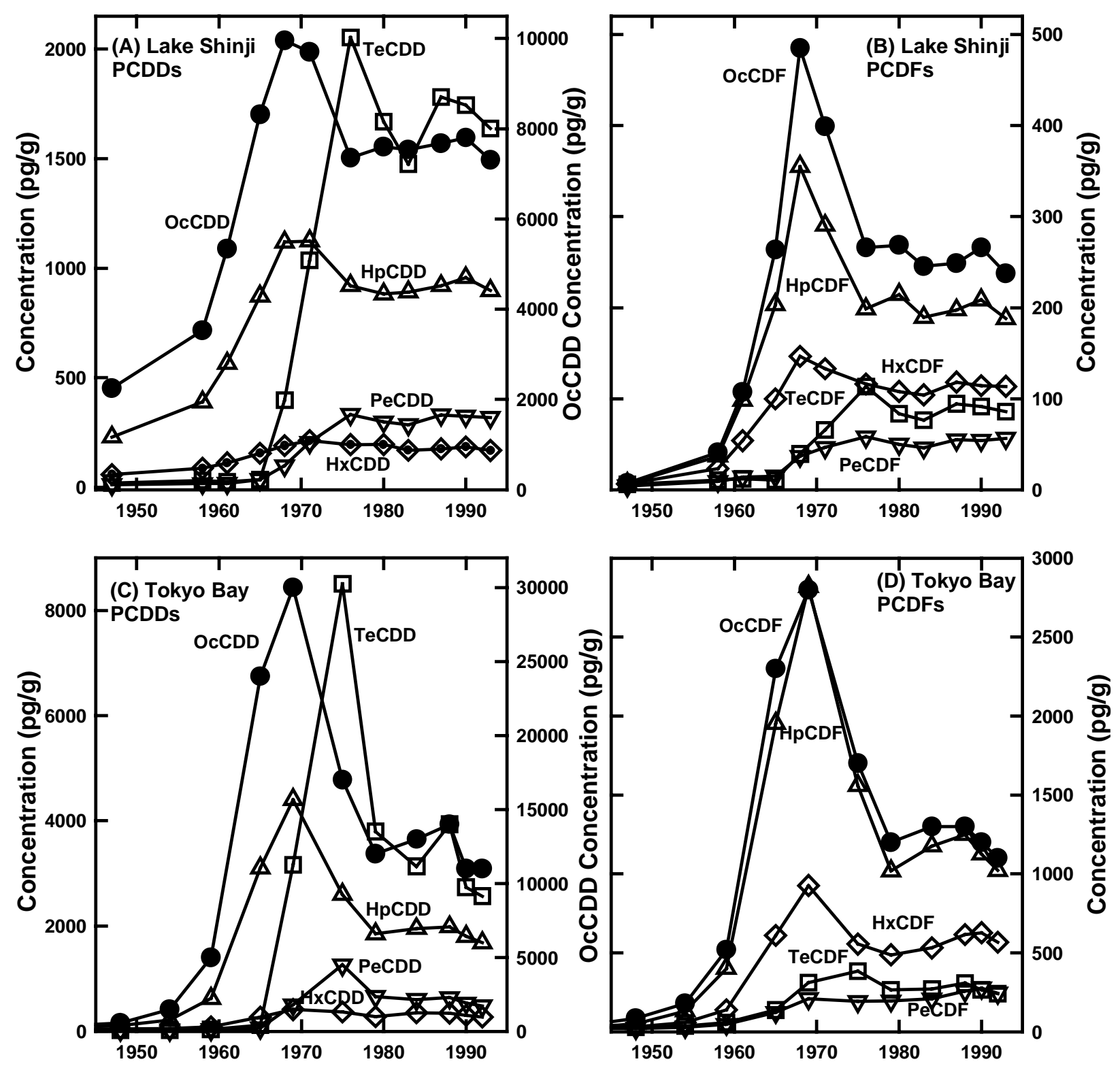

Figure S4. Time courses in the total concentration (pg/g) of each homologue in Lake Shinji (AB) and Tokyo Bay (C-D). In A and C, right y-axis is for OcCDD and left y-axis is for all other homologues. 
V. Kinetics of Dechlorination Pathways
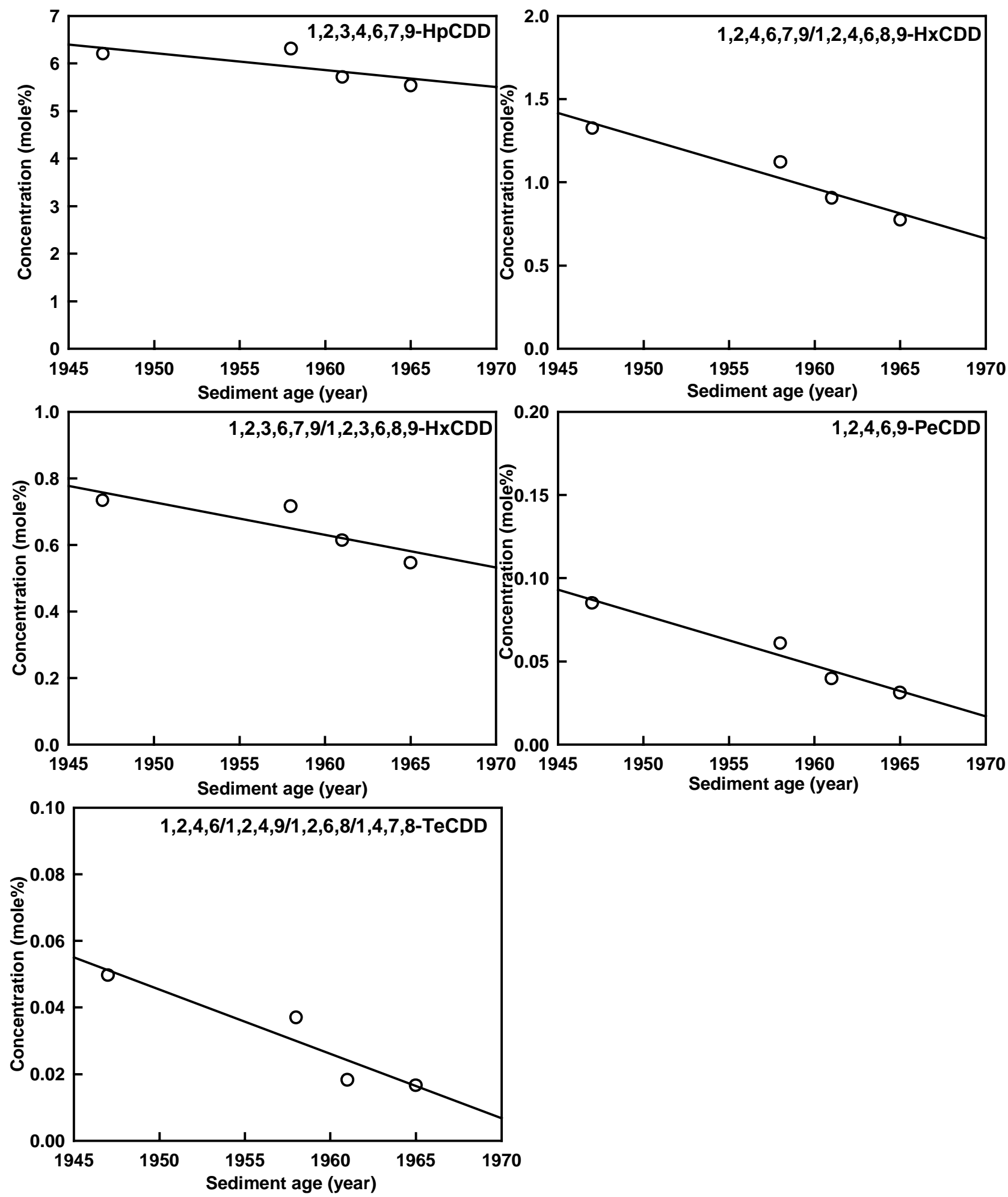

Figure S5A. Linear regression of the concentration (mole\% of total PCDD/Fs) versus sediment age (year) for the main PCDD congeners of the dechlorination fingerprint in Lake Shinji. 

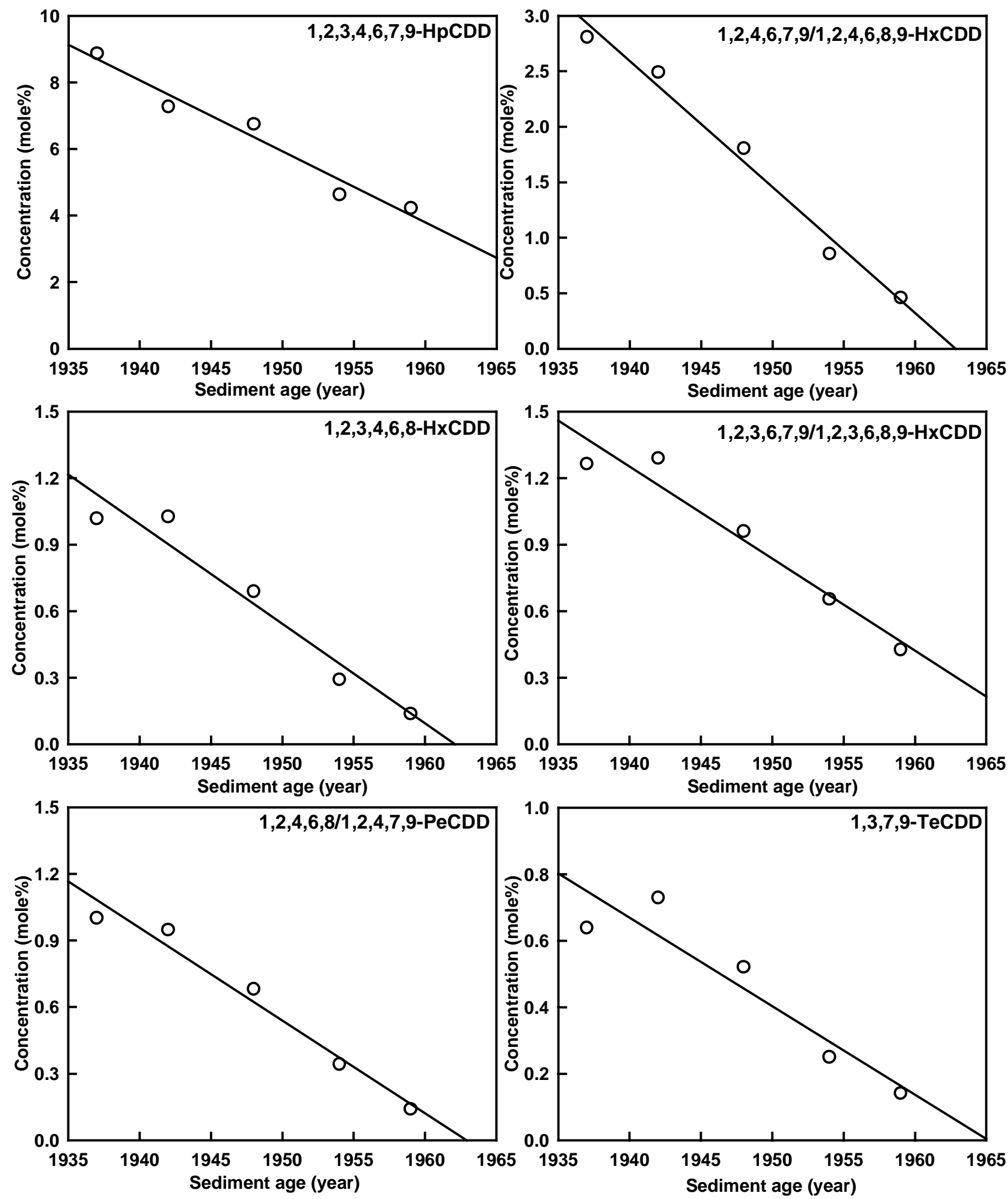

Figure S5B. Linear regression of the concentration (mole\% of total PCDD/Fs) versus sediment age (year) for the main PCDD congeners of the dechlorination fingerprint in Tokyo Bay. 

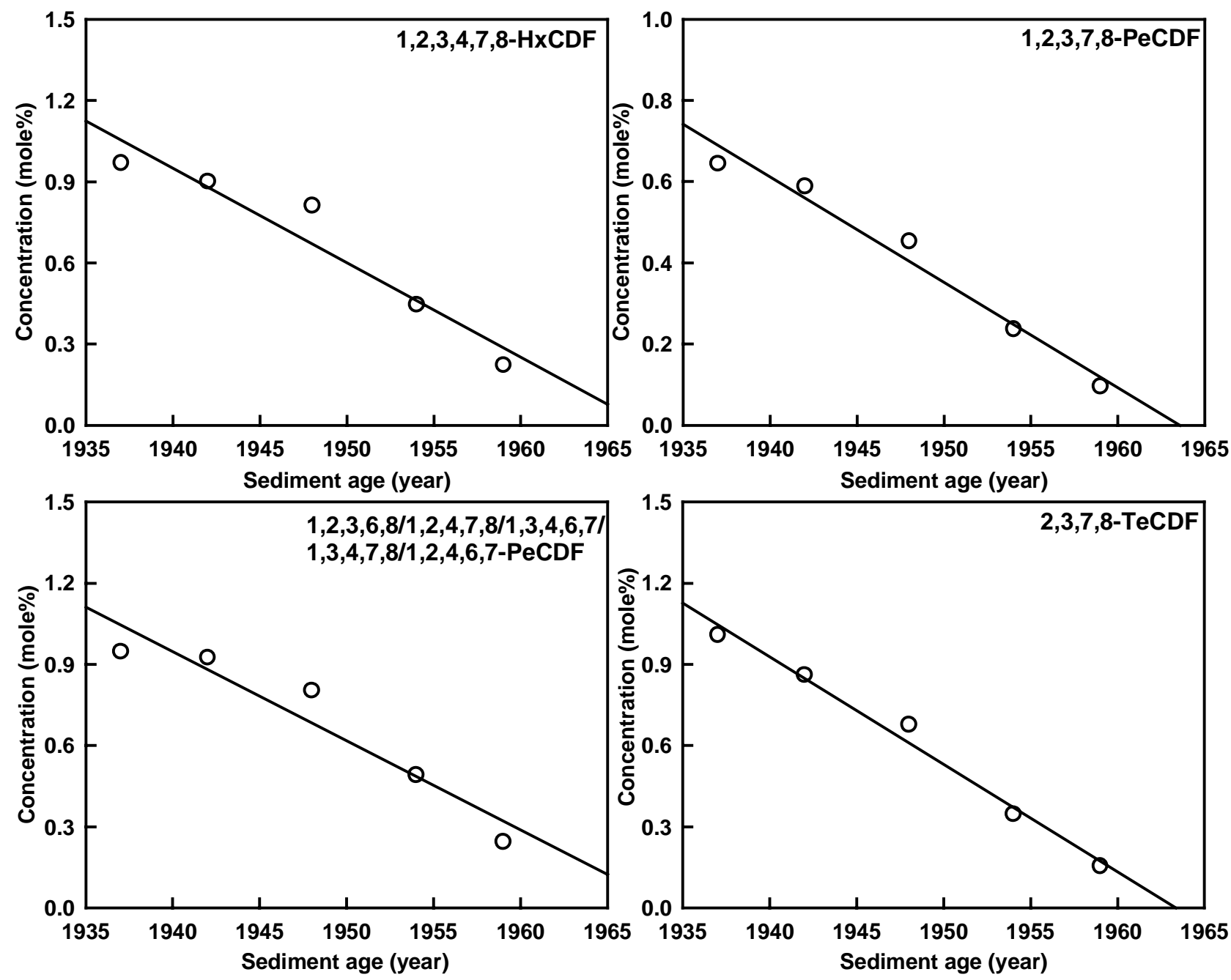

Figure S5C. Linear regression of the concentration (mole\% of total PCDD/Fs) versus sediment age (year) for the main PCDF congeners of the dechlorination fingerprint in Tokyo Bay. 


\section{Driving force for the Lateral Dechlorination of OcCDD}<smiles>CC(C)Cc1c(Cl)c(Cl)c2c(c1Cl)Oc1c(Cl)cc(Cl)c(Cl)c1Oc1ccc(Cl)c(Cl)c1O2</smiles>

Figure S6. Calculated partial charge on carbon atoms having chlorine substituents (most positive partial charge given in bold) and driving force for the dechlorination of each molecule (values beside each arrow; $\Delta \mathrm{G}$ in $\mathrm{kJ} / \mathrm{mol}$ ). Figure adopted from (6).

\section{References}

(1) Masunaga, S.; Takasuga, T.; Nakanishi, J. Dioxin and dioxin-like PCB impurities in some Japanese agrochemical formulations. Chemosphere 2001, 44, 873-885.

(2) Masunaga, S.; Nakanishi, J. Dioxin impurities in old Japanese agrochemical formulations. Organohalogen Compd. 1999, 41, 41-44.

(3) Ogura, I.; Masunaga, S.; Nakanishi, J. Congener-specific characterization of PCDDs/PCDFs in atmospheric deposition: comparison of profiles between deposition, source and environmental sink. Chemosphere 2001, 45, 173-183. 
(4) "Dioxins Emission Inventory," Environmental Agency of Japan, 2000 (in Japanese).

(5) Ogura, I.; Masunaga, S.; Nakanishi, J. Atmospheric deposition of polychlorinated dibenzo-pdioxins, polychlorinated dibenzofurans, and dioxin-like polychlorinated biphenyls in the Kanto Region, Japan. Chemosphere 2001, 44, 1473-1487.

(6) Lynam, M. M.; Kuty, M.; Damborsky, J.; Koca, J.; Adriaens, P. Molecular orbital calculations to describe microbial reductive dechlorination of polychlorinated dioxins. Environ.

Toxicol. Chem. 1998, 17, 988-997. 